\title{
The complete genome sequence of a bile-isolated Stenotrophomonas maltophilia ZT1
}

\author{
Min Zhang ${ }^{1}$, Lixiang Li ${ }^{2}$, Hongwei $\operatorname{Pan}^{3}$ and Tao Zhou ${ }^{1^{*}}$ (1)
}

\begin{abstract}
Background: Stenotrophomonas maltophilia is one of the most frequently isolated opportunistic pathogens that can cause infections in humans. Many researches concerned the mechanism of antibiotic resistance displayed by S. maltophilia, however, the mechanism of its pathogenesis and its adaptation to special niches, such as bile, remain unclear.

Results: In this study, the S. maltophilia strain ZT1 was isolated from human bile. Its genome was sequenced and a circular chromosome of 4,391,471 bp was obtained with a GC content of $66.51 \%$. There were 3962 protein-coding sequences, 7 rRNAs and 74 tRNAs in the chromosome. Compared with Virulence Factor Database, we identified more than 500 candidate virulence genes including genes encoding fimbrial assembly protein, enterobactin synthesis pathway proteins, efflux pumps, and the DNA and/or proteins secretion system in the genome of strain ZT1. Additionally, there were at least 22 genes related to bile adaption, including emrAB, acrRAB, galU, rfbC, to/C and $m d t A B C$.

Conclusions: This is the first study to reveal the whole genome sequence of the ZT1 strain of S. maltophilia isolated from human bile. We identified hundreds virulence factors and 22 bile adaptation-related genes in the genome of the S. maltophilia strain ZT1. Further comparative genomic analysis and functional verification would aid in understanding the pathogenesis and bile adaptation of S. maltophilia.
\end{abstract}

\section{Background}

Stenotrophomonas maltophilia is the third largest nonfermentative gram-negative bacillus, after Pseudomonas aeruginosa and Acinetobacter baumannii [1]. It can cause opportunistic infections, especially in hospitalized and immunocompromised patients. In recent year, the number of clinical isolates of $S$. maltophilia has shown an upward trend [2]. Many clinical studies have found that $S$. maltophilia strains are resistant to several antibiotics, making its treatment challenging and infection lifethreatening [3]. Currently, S. maltophilia is considered an

*Correspondence: zhoutao@sdu.edu.cn

${ }^{1}$ Department of Gastroenterology, Qilu Hospital, Shandong University, 107 Wenhuaxi Road, Shandong 250012 Jinan, People's Republic of China

Full list of author information is available at the end of the article emerging multidrug-resistant, global, and opportunistic pathogen [4].

S. maltophilia is a common microbe widely distributed in water, soil, plant-associated habitats, and animal tissues [5]. S. maltophilia has been isolated from the sputum, secretion, urine, blood, wound drainage and cerebrospinal fluid of patients [1]. These studies demonstrate the high environmental adaptability of $S$. maltophilia [2]. Currently, there are more than 600 genome assemblies of S. maltophilia accessible in the NCBI database. Many genomic studies have focused on the genes related to antibiotic resistance and the virulence of $S$. maltophilia [6-8]. However, few studies have investigated the genetic mechanism for environmental adaptation of S. maltophilia.

S. maltophilia is also a common bacterium in bile [9]. However, no study has focused on the genome of original author(s) and the source, provide a link to the Creative Commons licence, and indicate if changes were made. The images or other third party material in this article are included in the article's Creative Commons licence, unless indicated otherwise in a credit line to the material. If material is not included in the article's Creative Commons licence and your intended use is not permitted by statutory regulation or exceeds the permitted use, you will need to obtain permission directly from the copyright holder. To view a copy of this licence, visit http://creativecommons.org/licenses/by/4.0/. The Creative Commons Public Domain Dedication waiver (http://creativeco mmons.org/publicdomain/zero/1.0/) applies to the data made available in this article, unless otherwise stated in a credit line to the data. 
bile-isolated S. maltophilia strains. Here, we report the whole genome sequence of bile-isolated S. maltophilia strain ZT1. The bile adaptation and virulence-associated genes of the $S$. maltophilia strain ZT1 were also analyzed.

\section{Methods}

\section{Strain isolation and characterization}

A bile sample of a cholelithiasis patient was obtained for microbe culture and the S. maltophilia strain ZT1 was isolated on blood agar. This strain was then cultivated in brain-heart infusion broth for genomic DNA extraction under anaerobic conditions at $37{ }^{\circ} \mathrm{C}$ for $12 \mathrm{~h}$. The $16 \mathrm{~s}$ rRNA sequence was obtained by PCR using primers $27 \mathrm{~F}$ (5'-AGAGTTTGATCCTGGCTCAG-3') and 1492R (5'GGTTACCTTGTTACGACTT-3') and then sequenced. The obtained $16 \mathrm{~s}$ rRNA sequence was compared to the NCBI website using BLAST.

\section{Genome sequencing and de novo assembly}

A Bacteria DNA isolation kit (OMEGA Bio-Tek Inc., Norcross, GA, USA) was used to extract the genomic DNA from an overnight culture of the S. maltophilia strain ZT1. Subsequently, the quality of the genomic DNA was tested using a TBS-380 fluorometer (Turner BioSystems Inc., Sunnyvale, CA, USA). Then, a highly qualified DNA sample (OD260/280 $=1.8-2.0$, total amount $>6 \mu \mathrm{g}$ ) was utilized for genome sequencing. According to the sequencing protocol, approximately $3 \mu \mathrm{g}$ of genomic DNA was sequenced using an Illumina HiSeq Sequencer (Illumina Inc. San Diego, CA, USA) in the PE150 mode. Illumina pipeline CASAVA v1.8.2 (Illumina Inc. San Diego, CA, USA) was used for base calling to produce the raw sequencing data. Meanwhile, another $3 \mu \mathrm{g}$ of genomic DNA was used to generate a $20 \mathrm{~K}$ template library which was then sequenced using the PacBio RS Platform (Pacific Biosciences of California, Inc., Menlo Park, CA, USA).

The Illumina sequence data were used to establish the complexity of the genome as well as correct the PacBio long reads, as described previously [10]. First, genome assembly was performed using ABySS with multiple-Kmer parameters and then the optimal results was obtained for the assembly [11]. After that, the long reads generated by PacBio were assembled using Canu (https://github.com/marbl/canu). Then, the remaining local inner gaps were filled using GapCloser [12]. Finally, we corrected the single-base polymorphisms for the final assembly results.

Gene annotation was performed using the automatic prokaryotic genome annotation pipeline (https:// www.ncbi.nlm.nih.gov/genome/annotation_prok/). The CGView Server was used to create the circular genomic map of strain ZT1 [13]. B, The phylogenetic analysis was performed based on orthologous genes. First, OrthoMCL v2.0 was used to establish orthologous gene families with an E-value of $10^{-5}$ [14]. Second, the MUSCLE v3.8.31 software was used for multiple alignments [15]. Finally, phylogenetic analyses were performed using PhyML 3.0, based on maximum-likelihood (ML) methods. To calculate the bootstrap values, the GTR + G model was selected for ML analysis using 500 bootstrap replicates [16]. Several strains, including S. maltophilia NCTC10257 (LT906480.1), S. maltophilia SJTL3(CP029773.1), S. maltophilia Sm1 (GCA_001431665.1), S. maltophilia Sm2 (GCA_001651505.1), S. maltophilia Sm3 (GCA_001068765.1), S. maltophilia Sm4a (GCA_001069235.1), S. maltophilia Sm4b (GCA_000223885.1), S. maltophilia Sm5 (RAVG00000000), S. maltophilia Sm6 (GCA_002799015.1), S. maltophilia Sm7 (GCA_002798945.1), S. maltophilia Sm8 (RATO00000000), S. maltophilia Sm9 (GCA_001070945.1), S. maltophilia Sm10 (GCA_001297005.1), S. maltophilia Sm11 (GCA_002798885.1), S. maltophilia Sm12 (RAUV00000000), S. maltophilia Sm13 (ERR3300023), S. maltophilia Sm14 (GCA_002799245.1), S. maltophilia Sm15 (RATP0000000), S. maltophilia Sm16 (CP040439.1), S. maltophilia Sm17 (RAUD00000000), S. maltophilia Sm18 (GCA_002798925.1), S. maltophilia Sgn1 (GCA_002025605.1), S. maltophilia Sgn2 (GCA_002377295.1), S. maltophilia Sgn3 (GCA_000613205.1), S. maltophilia Sgn4 (GCA_001676435.1), S. bentonitica DSM103927 (GCA_013185915.1), S. nitritireducens 2001 (CP016756.1), S. acidaminiphila SUNEO (CP019797.1), S. rhizophila QL-P4 (CP016294.1); X. campestris NEB122 (CP051651.1), S. indicatrix DAIF1 (CP037883.1), S. lactitubi As-6 (GCA_016921095.1), and S. tumulicola JCM 30961 (GCA_014117215.1) were selected for phylogenetic tree analysis. The putative virulence-related genes in the genome of the S. maltophilia strain ZT1 were identified using the Virulence Factor Database (VFDB) with a score $>80$ and an e-value $<0.001$ [17].

\section{Results and discussion}

\section{General genome features of the S. maltophilia strain ZT1}

A total of $2712.2 \mathrm{Mb}$ data containing 18,081,966 raw reads were produced by the Illumina HiSeq Sequencer. After data filtering, 2611.8 Mb data containing $17,458,806$ high-quality reads were obtained. Meanwhile, $115,046(497.3 \mathrm{Mb})$ high-quality reads were produced by the Pac bio RS Platform with an average read length of 4322 bp. Sequence coverage was $>700$-fold. Furthermore, we assembled these reads and obtained a high-quality genome with only one scaffold in this study (Fig. 1). This 


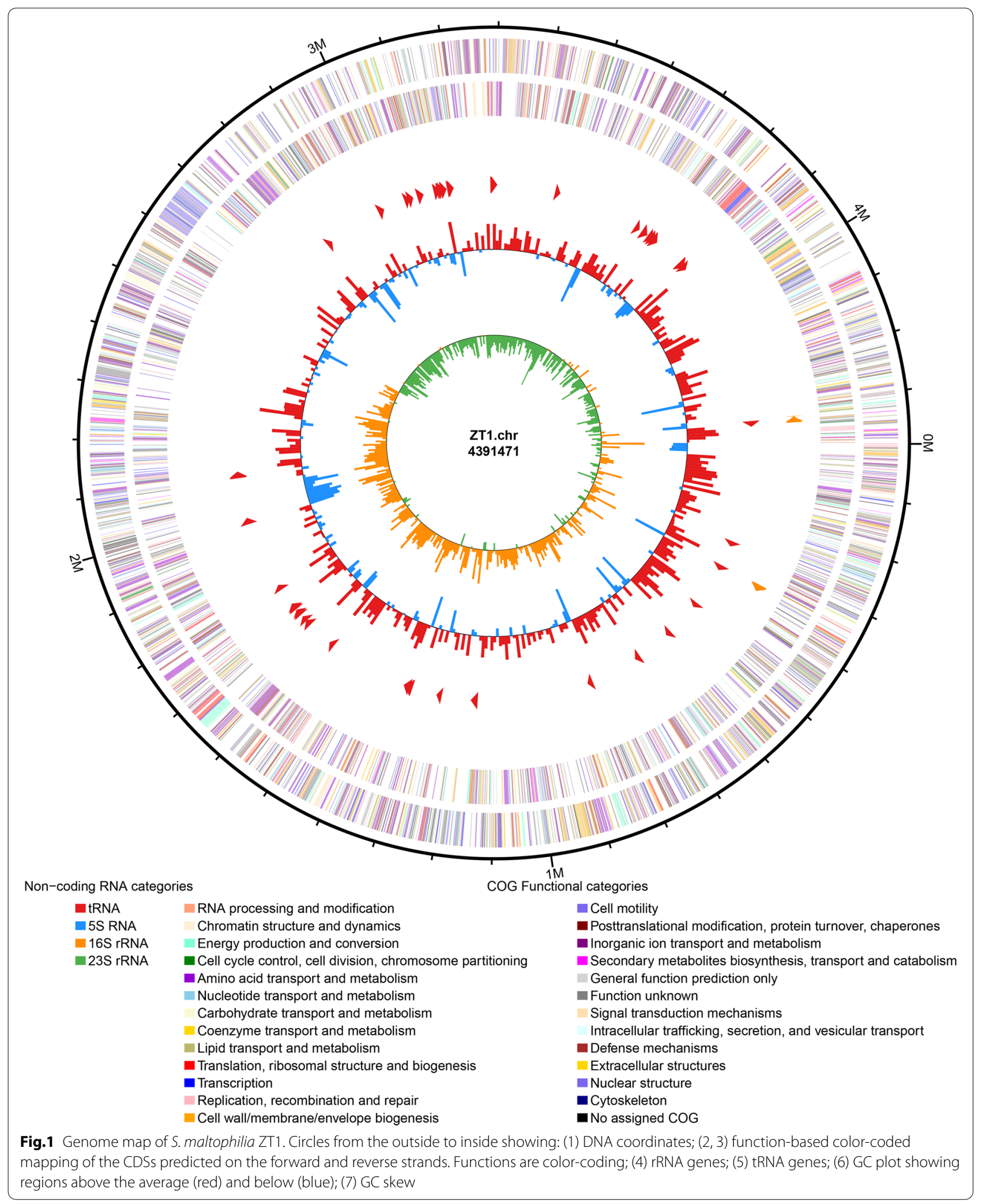


complete genome is $4.19 \mathrm{Mb}$ with a mean $\mathrm{G}+\mathrm{C}$ content of $66.51 \%$. After annotation, 3962 protein-coding sequences, seven rRNAs, and 74 tRNA genes were found in the chromosome of strain ZT1.

\section{Phylogenetic analysis}

The 16S rRNA gene sequence of strain ZT1 revealed the taxonomic status of strain ZT1 to be $S$. maltophilia by BLAST(data not shown). Phylogenetic analysis was carried out based on the whole genome DNA-sequence, and the results are shown in Fig. 2. A total of 24 type strains of S. maltophilia, including S. maltophilia NCTC10257 (LT906480.1), S. maltophilia SJTL3(CP029773.1), S. maltophilia sm5 (RAVG00000000), were selected as standard. It was found that the strain ZT1 was most closely related to the S. maltophilia sm5 clade.

\section{Identification of genes related to bile adaption}

Bile acids are a group of steroids with high stability and biocompatibility. One of the major physiological roles is antimicrobial activity, as they have a structure similar to the antimicrobial peptides [18]. S. maltophilia strain ZT1 could live in bile, and which indicates an inherent tolerance to high concentrations of bile. Genomic analysis of the S. maltophilia strain ZT1 showed the presence of 22 genes related to bile adaptation (Table 1). Half of them were genes encoding efflux pumps, including emrAB, acr $A B$, tolC, mexAB, mdt $A B C$, and porin $\mathrm{F}$. We also identified other genes, such as $\mathrm{O}$-antigen biosynthesis-related (rfbABC), DNA binding protein (hupB), DNA mismatch repair protein (mutS), and zinc uptake regulation protein (zur) genes, which are related to bile adaption.

\section{Analysis of virulence associated genes}

S. maltophilia is a conditional pathogen and strain ZT1 was isolated from a patient with cholelithiasis. Therefore, analysis of putative virulence-associated genes in the genome of strain ZT1 was performed by aligning gene sequences to VFDB. The results indicated that more than 500 hundred virulence-associated genes were predicted in the genome of strain ZT1 (Additional file 1: Table S1). We found 88 gene related to flagellar biosynthesis in the genome of strain ZT1; additionally, there was a flagellar encoding gene island from ZT1002347 to ZT1002401. The virulence-associated fimbrial assembly

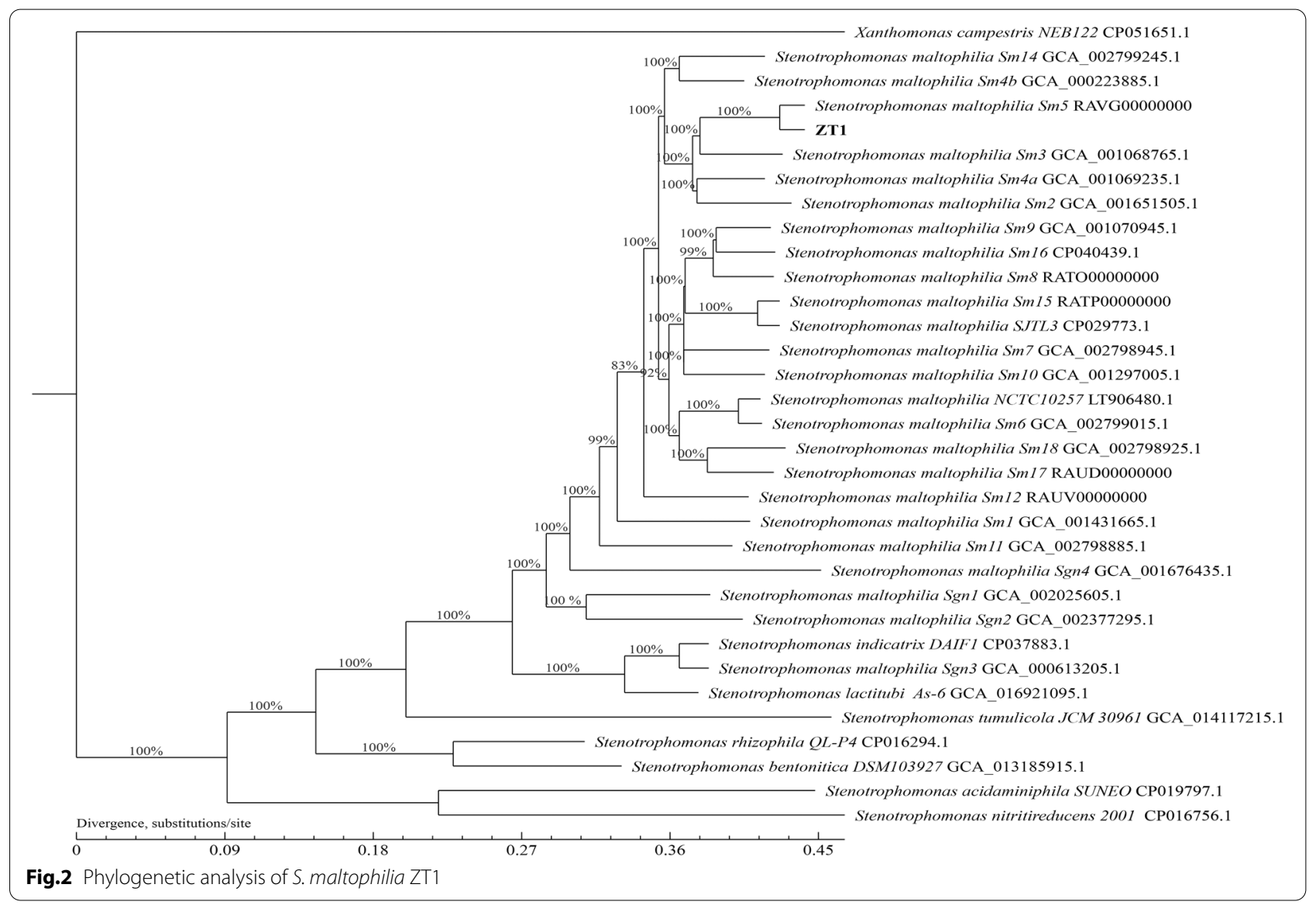


Table 1 The genes related to bile adaption in S. maltophilia ZT1

\begin{tabular}{|c|c|c|c|}
\hline Gene ID & Swissprot_tophit_descrip & $\begin{array}{l}\text { Swissprot top } \\
\text { similarity \% }\end{array}$ & KEGG gene name \\
\hline ZT1002978 & Multidrug export protein EmrB OS=Escherichia coli (strain K12) & 49.4 & emrB \\
\hline ZT1002979 & Multidrug export protein EmrA OS= Haemophilus influenzae (strain ATCC 51907) & 45.2 & emrA \\
\hline ZT1000837 & HTH-type transcriptional regulator AcrR OS=Shigella flexneri & 31.4 & acrR, smeT \\
\hline ZT1000838 & Multidrug efflux pump subunit AcrA OS= Escherichia coli (strain K12) & 43.9 & acrA, mexA \\
\hline ZT1000839 & Multidrug efflux pump subunit AcrB OS= Escherichia coli (strain K12) & 64 & $\mathrm{acr} B, \operatorname{mexB}$ \\
\hline ZT1000961 & Outer membrane protein TolC OS=Vibrio cholerae serotype O1 (strain ATCC 39,315) & 31.7 & tolC \\
\hline ZT1001690 & Multidrug efflux pump subunit AcrB OS = Escherichia coli (strain K12) & 50 & $\mathrm{acr} B, \operatorname{mexB}$ \\
\hline ZT1001691 & Multidrug efflux pump subunit AcrA OS=Escherichia coli (strain K12) & 41.2 & acrA, mexA \\
\hline ZT1003728 & O-antigen biosynthesis protein $\mathrm{RfbC} O \mathrm{OS}=$ Myxococcus xanthus & 42.1 & $\mathrm{rfbC}$ \\
\hline ZT1003730 & O-antigen export system ATP-binding protein $\mathrm{RfbB} O \mathrm{OS}=$ Myxococcus xanthus & 39 & ABC-.LPSE.A \\
\hline ZT1003731 & O-antigen export system permease protein RfbA OS=Klebsiella pneumoniae & 22 & - \\
\hline ZT1000559 & Virulence transcriptional regulatory protein PhoP OS = Salmonella typhi & 39.4 & - \\
\hline ZT1000233 & Virulence transcriptional regulatory protein PhoP OS = Salmonella typhi & 43.7 & phoP \\
\hline ZT1000632 & Multidrug resistance protein MdtC OS=Enterobacter sp. (strain 638) & 44.5 & - \\
\hline ZT1000633 & Multidrug resistance protein MdtB OS=Xenorhabdus bovienii (strain SS-2004) & 51.1 & mdtB \\
\hline ZT1000634 & Multidrug resistance protein MdtA OS= Dickeya zeae (strain Ech586) & 40.3 & mdtA \\
\hline ZT1003430 & Outer membrane porin F OS = Pseudomonas aeruginosa (strain ATCC 15,692) & 32 & TC.OOP \\
\hline ZT1003393 & DNA-binding protein HU OS=Xylella fastidiosa (strain Temecula1 / ATCC 700,964) & 76.4 & hupB \\
\hline ZT1002604 & UTP-glucose-1-phosphate uridylyltransferase OS $=$ Pseudomonas aeruginosa (strain PAO1) & 46.5 & galU, galF \\
\hline ZT1002538 & UDP-glucose 4-epimerase OS= Bacillus halodurans (strain ATCC BAA-125) & 51.4 & galE, GALE \\
\hline ZT1003099 & DNA mismatch repair protein MutS OS=Xanthomonas campestris (strain ATCC 33,913) & 89.6 & muts \\
\hline ZT1003056 & Zinc uptake regulation protein OS $=$ Shigella flexneri & 37.6 & zur \\
\hline
\end{tabular}

protein-encoding genes included pilB, pilQ, pilJ, pilT, pilC, pilU, pilM, pilD, flhA, mrkC, fauA, and flgI. Enterobactin is a virulence factor of $S$. maltophilia [19]. An entCEBFA operon (ZT1002005-ZT1002010) containing genes encoding for proteins of the enterobactin synthesis pathway as well as an enterobactin exporter encoding gene entS (ZT1001564) were found. The efflux pumpassociated virulence factors are listed in Additional file 2: Table S2. Several RND family multidrug efflux pumps, NodT family efflux transporter, multidrug ABC transporter and other efflux pumps were found in the genome of strain ZT1, which may be related to antibiotic resistance in S. maltophilia. The DNA and/or protein secretion system is another type of virulence factor in $S$. maltophilia [20]. In the genome of strain ZT1, we found two independent type II secretion system operons, one containing 11 genes (xpsDNMLKJIHGFE; ZT1003667ZT1003677) and the second contained at least another 11 gene (gspGKLMDEFJIHC, ZT1002072-ZT1002085). Some of these were identified as virulence factors, as shown in Additional file 3: Table S3. Meanwhile, a type IV secretion system operon was also found in the genome of strain ZT1, containing genes encoding for virB4, virB8, virB11. These virulence factors might contribute $S$. maltophilia infection and require further investigation.

\section{Supplementary Information}

The online version contains supplementary material available at https://doi. org/10.1186/s13099-021-00456-y.

Additional file 1. Table S1. The results indicated that more than 500 hundred virulence-associated genes were predicted in the genome of strain ZT1.

Additional file 2. Table S2. The efflux pump associated virulence factor in S. maltophilia ZT1 predicted by VFDB.

Additional file 3. Table S3. The secretion system associated virulence factor in S. maltophilia ZT1 predicted by VFDB.

\section{Acknowledgements}

We would like to thank Editage (www.editage.cn) for English language editing

\section{Authors' contributions}

$T Z$ and $L X L$ designed the study; $M Z$ and $L X L$ isolated and identified the $S$. maltophilia strain $Z \mathrm{~T} 1 ; \mathrm{MZ}$ and $\mathrm{LXL}$ analyzed data and wrote the manuscript; HWP revised the manuscript. LXL and TZ support this study the manuscript. All authors read and approved the final manuscript.

\section{Funding}

This research was supported by the Key Research and Development Program of Shandong Province (2019GHZ022 and 2019GSF108254).

\section{Availability of data and materials}

The completed genome sequence of S. maltophilia strain ZT1 has been deposited into GenBank database with accession number CP071784. 


\section{Declarations}

\section{Ethics approval and consent to participate}

This study was approved by the ethics committees in Qilu hospital of Shandong University.

\section{Competing of interests}

The authors declare that they have no competing interests.

\section{Author details}

${ }^{1}$ Department of Gastroenterology, Qilu Hospital, Shandong University, 107 Wenhuaxi Road, Shandong 250012 Jinan, People's Republic of China. ${ }^{2}$ Key Laboratory of Translational Gastroenterology, Shandong University, 250012 Jinan, People's Republic of China. ${ }^{3}$ Department of Clinical Laboratory, Qilu Hospital, Shandong University, Shandong 250012 Jinan, China.

Received: 23 March 2021 Accepted: 6 October 2021

Published online: 28 October 2021

\section{References}

1. Hu LF, Xu XH, Li HR, Gao LP, Chen X, Sun N, Liu YY, Ying HF, Li JB. Surveillance of antimicrobial susceptibility patterns among Stenotrophomonas maltophilia isolated in China during the 10-year period of 2005-2014. J Chemother. 2018;30(1):25-30.

2. Adegoke AA, Stenstrom TA, Okoh Al. Stenotrophomonas maltophilia as an emerging ubiquitous pathogen: looking beyond contemporary antibiotic therapy. Front Microbiol. 2017;8:2276.

3. Alsuhaibani M, Aljarbou A, Althawadi S, Alsweed A, Al-Hajjar S. Stenotrophomonas maltophilia bacteremia in children: risk factors and mortality rate. Antimicrob Resist Infect Control. 2021;10(1):19.

4. Brooke JS. Stenotrophomonas maltophilia: an emerging global opportunistic pathogen. Clin Microbiol Rev. 2012;25(1):2-41.

5. Pinski A, Zur J, Hasterok R, Hupert-Kocurek K. Comparative genomics of Stenotrophomonas maltophilia and Stenotrophomonas rhizophila revealed characteristic features of both species. Int J Mol Sci. 2020. https://doi.org/ 10.3390/ijms21144922.

6. Yero D, Huedo P, Conchillo-Sole O, Martinez-Servat S, Mamat U, Coves X Llanas F, Roca I, Vila J, Schaible UE, et al. Genetic variants of the DSF quorum sensing system in Stenotrophomonas maltophilia influence virulence and resistance phenotypes among genotypically diverse clinical isolates. Front Microbiol. 2020;11:1160.

7. Han L, Zhang RM, Jia L, Bai SC, Liu XW, Wei R, Cui CY, Sun RY, Wang MG, Cai DT, et al. Diversity of L1/L2 genes and molecular epidemiology of high-level carbapenem resistance Stenotrophomonas maltophilia isolates from animal production environment in China. Infect Genet Evol. 2020;86:104531.
8. Esposito A, Pompilio A, Bettua C, Crocetta V, Giacobazzi E, Fiscarelli E, Jousson O, Di Bonaventura G. Evolution of Stenotrophomonas maltophilia in cystic fibrosis lung over chronic infection: a genomic and phenotypic population study. Front Microbiol. 2017;8:1590.

9. Kaya M, Bestas R, Bacalan F, Bacaksiz F, Arslan EG, Kaplan MA. Microbial profile and antibiotic sensitivity pattern in bile cultures from endoscopic retrograde cholangiography patients. World J Gastroenterol. 2012;18(27):3585-9.

10. Yu T, Li L, Zhao Q, Wang P, Zuo X. Complete genome sequence of bileisolated Enterococcus avium strain 352. Gut Pathog. 2019;11:16.

11. Jackman SD, Vandervalk BP, Mohamadi H, Chu J, Yeo S, Hammond SA Jahesh G, Khan H, Coombe L, Warren RL, et al. ABySS 2.0: resourceefficient assembly of large genomes using a Bloom filter. Genome Res. 2017;27(5):768-77.

12. Luo R, Liu B, Xie Y, Li Z, Huang W, Yuan J, He G, Chen Y, Pan Q, Liu Y, et al. SOAPdenovo2: an empirically improved memory-efficient short-read de novo assembler. Gigascience. 2012;1 (1):18.

13 Grant JR, Stothard P. The CGView server: a comparative genomics tool for circular genomes. Nucleic Acids Res. 2008. https://doi.org/10.1093/nar/ gkn179.

14. Li L, Stoeckert CJ Jr, Roos DS. OrthoMCL: identification of ortholog groups for eukaryotic genomes. Genome Res. 2003;13(9):2178-89.

15. Edgar RC. MUSCLE: multiple sequence alignment with high accuracy and high throughput. Nucleic Acids Res. 2004;32(5):1792-7.

16 Guindon S, Dufayard JF, Lefort V, Anisimova M, Hordijk W, Gascuel O. New algorithms and methods to estimate maximum-likelihood phylogenies: assessing the performance of PhyML 3.0. Syst Biol. 2010;59(3):307-21.

17. Chen L, Zheng D, Liu B, Yang J, Jin Q. VFDB 2016: hierarchical and refined dataset for big data analysis-10 years on. Nucleic Acids Res. 2016;44(D1):D694-697.

18 di Gregorio MC, Cautela J, Galantini L. Physiology and physical chemistry of bile acids. Int J Mol Sci. 2021. https://doi.org/10.3390/ijms22041780.

19. Kalidasan V, Joseph N, Kumar S, AwangHamat R, Neela VK. iron and virulence in Stenotrophomonas maltophilia: all we know so far. Front Cell Infect Microbiol. 2018:8:401.

20. Nas MY, White RC, DuMont AL, Lopez AE, Cianciotto NP. Stenotrophomonas maltophilia encodes a VirB/NirD4 type IV secretion system that modulates apoptosis in human cells and promotes competition against heterologous bacteria including Pseudomonas aeruginosa. Infect Immun. 2019. https://doi.org/10.1128/IAl.00457-19.

\section{Publisher's Note}

Springer Nature remains neutral with regard to jurisdictional claims in published maps and institutional affiliations.

Ready to submit your research? Choose BMC and benefit from:

- fast, convenient online submission

- thorough peer review by experienced researchers in your field

- rapid publication on acceptance

- support for research data, including large and complex data types

- gold Open Access which fosters wider collaboration and increased citations

- maximum visibility for your research: over 100M website views per year

At $B M C$, research is always in progress.

Learn more biomedcentral.com/submissions 